\title{
BMJ Open Scoping review protocol: effectiveness of individualised nutritional care plans to reduce malnutrition during hospitalisation and up to 3 months after discharge
}

\author{
Kari Ingstad, ${ }^{1}$ Lisbeth Uhrenfeldt, ${ }^{2}$ Ingjerd Gåre Kymre, ${ }^{2}$ Conni Skrubbeltrang, ${ }^{3}$ \\ Preben Ulrich Pedersen ${ }^{4}$
}

To cite: Ingstad K, Uhrenfeldt L, Kymre IG, et al. Scoping review protocol: effectiveness of individualised nutritional care plans to reduce malnutrition during hospitalisation and up to 3 months after discharge. BMJ Open 2019;9:e032615. doi:10.1136/ bmjopen-2019-032615

- Prepublication history and additional material for this paper are available online. To view these files, please visit the journal online (http://dx.doi. org/10.1136/bmjopen-2019032615).

Received 27 June 2019 Revised 09 August 2019 Accepted 22 August 2019
Check for updates

(C) Author(s) (or their employer(s)) 2019. Re-use permitted under CC BY-NC. No commercial re-use. See rights and permissions. Published by BMJ.

For numbered affiliations see end of article.

Correspondence to

Kari Ingstad;

kari.ingstad@nord.no

\section{ABSTRACT}

Introduction More than $20 \%$ of patients are malnourished after hospitalisation. Malnutrition may negatively impact patients' outcomes as it increases the risk of complications, morbidity, mortality and loss of function. However, hospital-initiated transitional care can improve some outcomes in hospitalised adult patients. The objective of this scoping review is to map the literature that assesses the effectiveness of individualised nutritional care plans to reduce malnutrition during hospitalisation and for the first 3 months after discharge.

Methods and analysis This protocol is based on the framework outlined by Arksey and 0'Malley. The search strategy was developed by a medical librarian. We will search for relevant literature from the following databases: MEDLINE via PubMed, Cumulative Index to Nursing and Allied Health Literature, and Embase. We will also search the reference lists of included studies. Two independent reviewers will screen abstracts and full articles in parallel, from the included studies using specific inclusion and exclusion criteria. The Preferred Reporting Items for Systematic Reviews and Meta-Analysis Protocols checklist facilitated the preparation of this research protocol. The scoping review will provide a narrative account of the findings from the existing literature through thematic content analysis of the extracted literature.

Ethics and dissemination Since all data will be obtained from publicly available materials, research ethics approval is not required for this scoping review. The research findings will be submitted for publication in a relevant open-access peer-reviewed journal and presented at relevant conferences.

\section{INTRODUCTION}

The prevention of malnutrition is a common problem in hospitals worldwide, especially among older hospitalised adults. ${ }^{1-4}$ Different studies have estimated the prevalence of malnutrition to be $20 \%-50 \%$ at the time of discharge. ${ }^{5-7}$ Poor nutrition care may adversely impact patients' outcomes as
Strengths and limitations of this study

- This will be the first scoping review to identify and map studies that assess the effectiveness of individualised nutritional care plans.

- The evidence obtained from the included studies, when summarised, will help to guide nutritional practice and future research.

- The review will have no language restrictions; studies published in languages other than English, German, Danish, Swedish and Norwegian will be translated using Google Translate.

malnutrition increases their risks of complications, morbidity, mortality and loss of function. ${ }^{6-12}$

Nutritional issues or malnutrition neither occur nor are solved overnight. Communication and collaboration among different healthcare providers are of utmost importance to ensure that patients receive coherent and coordinated nutritional care and treatment. $^{13}$

In most countries, the average length of stay in hospitals has declined since $2000 .{ }^{14}$ In 2015, the average length of stay in hospitals for all causes among the 36 member countries belonging to the Organisation for Economic Co-operation and Development was approximately 8 days. ${ }^{14} 15$ With shorter hospital stays, there will be an increased need for nutrition services after discharge to support improvements in nutritional status to prevent complications and re-admission. ${ }^{16-18}$ However, lack of communication and continuity in care has been identified in several studies as a major risk factor for malnutrition. 131920

A systematic review conducted in 2012 concluded that transitional care initialised in hospitals could yield positive outcomes 
in hospitalised adult patients. ${ }^{21}$ One study showed that dietetic care in hospitals was the most influential predictor of post-hospital care.$^{22}$ Early and extended nutrition intervention can improve nutrition status and reduce length of hospital stay. ${ }^{23}$ Recent studies have found that hospital-initiated care plans with follow-up by competent healthcare staff at home might be effective for improving nutritional status, as well as for reducing complications and re-admission rates, among geriatric patients. ${ }^{24}{ }^{25}$ The benefits of nutritional intervention initiated during hospital stay may be lost if continuity of care is not adequately addressed at the time of discharge. Therefore, individualised care plans with follow-ups might be an effective intervention strategy to improve nutritional care.

Individualised care plans are developed based on an individual assessment of patients' nutritional needs, conditions and desires. ${ }^{26} \mathrm{~A}$ nutrition care plan documents and incorporates the recommendations made by the multidisciplinary team involved in the patient's care. This can include, but is not limited to, recommendations made by dietitians, speech pathologists, occupational therapists, nurses, midwives and the medical team. ${ }^{27} \mathrm{~A}$ nutrition care plan contains clearly documented nutrition interventions to attain identified goals of treatment; these plans are reviewed regularly and are documented to reflect changes. ${ }^{26} 27$

There is a need to synthesise research evidence on this subject. Scoping reviews have great utility for mapping research evidence and are often used to identify gaps in the evidence, clarify key concepts and report on the types of evidence that address and inform practice in a given field. ${ }^{28}$ The scoping review may be followed by a systematic review if it is concluded that a large body of evidence exists in the field. ${ }^{29}$ The time frame might be expanded in the potential systematic review.

A preliminary search of the literature, including that from the Joanna Briggs Institute Database of Systematic Reviews and Implementation Reports, Cochrane Database of Systematic Reviews, PROSPERO International Prospective Register of Systematic Reviews, MEDLINE via PubMed, and Cumulative Index to Nursing and Allied Health Literature (CINAHL), was performed between April and July 2019. No existing reviews similar to the proposed scoping review were identified.

\section{Study objective}

This study aims to identify and map studies that assess the effectiveness of individualised nutritional care plans to reduce malnutrition during hospital stay and for the first 3 months after discharge from the hospital.

\section{METHODS AND ANALYSIS}

This proposed scoping review will be guided by Arksey and O'Malley's scoping review framework, ${ }^{30}$ which has been further developed by Levac et al and the Joanna Briggs Institute. ${ }^{31} 32$ Briefly, the framework includes five stages: (1) identifying a research question, (2) identifying relevant studies, (3) study selection, (4) charting the data and (5) collating, summarising and reporting the results. ${ }^{30}$ Each stage is discussed in detail below. The PRISMA-ScR (Preferred Reporting Items for Systematic reviews and Meta-Analyses Extension for Scoping Reviews): Checklist and Explanation ${ }^{33}$ facilitated the preparation of this research protocol.

\section{Stage 1: identifying the research question}

The objective of this scoping review is to map studies that assess the effectiveness of individualised nutritional care plans, for patients who have undergone a transition from hospital. Prior to identifying the research question, an exploratory review of the literature on malnutrition during hospital stay helped to refine the scope of the present protocol. During the preliminarily exploratory review, it became clear that hospitals worldwide are struggling to prevent malnutrition. Transitional care that promotes nutritional care is necessary to achieve shorter hospital stays, as transitional care initialised in the hospital yields positive outcomes in hospitalised adult patients.

Based on the initial exploratory research, the following research questions were identified:

1. What types of individualised nutritional care plan intervention are addressed in the literature?

2. What outcomes have been measured in the studies?

3. Are these interventions effective?

4. If these interventions are effective, in what way are they effective?

5. What populations have been studied?

This review seeks to establish an understanding of if and how individualised nutritional care plans can prevent malnutrition.

\section{Stage 2: identifying relevant studies}

The search strategy was developed by the research team in collaboration with an experienced medical librarian (CS). Databases to be searched as part of this review include MEDLINE via PubMed, CINAHL and Embase. The databases will be searched from the time of database inception. The keywords to be used during the initial search, as well as the strategy for searching MEDLINE via PubMed, are shown in online supplementary appendix 1. Literature search strategies will be developed using medical subject headings and text words related to the population, concept and context of the study, ${ }^{26}$ as shown in table 1.

This scoping review will include primary research articles published in peer-reviewed journals, review articles and open grey literature that address the research question. The review will consider both experimental and quasi-experimental studies, including randomised controlled trials, non-randomised controlled trials, before-and-after studies and interrupted time-series studies. In addition, analytical observational studies, including prospective and retrospective cohort studies, case-control studies and analytical cross-sectional studies, will be considered for inclusion. This review will also 
Table 1 PCC grid showing identified search terms with truncated keywords and MeSH terms for the MEDLINE search via PubMed

\begin{tabular}{|c|c|c|c|}
\hline & Population & Concept & Context \\
\hline Text words & $\begin{array}{l}\text { Patient } \\
\text { Elder }^{\star} \\
\text { Older* }^{\star} \\
\text { Senior* } \\
\text { Nutritional risk }\end{array}$ & $\begin{array}{l}\text { Nutrition } \\
\text { Malnourished } \\
\text { Undernutrition } \\
\text { Nutritional care plan* } \\
\text { Nutritional hand over* } \\
\text { Discharge plan } \\
\text { Malnutrition prevention } \\
\text { Nutritional intervention } \\
\text { Individualised nutritional care plan }\end{array}$ & $\begin{array}{l}\text { Transition* } \\
\text { Transfer }^{\star} \\
\text { Discharge }^{\star} \\
\text { Hand over } \\
\text { Patient handoff* }\end{array}$ \\
\hline MEDLINE & Patients (MeSH) & $\begin{array}{l}\text { Malnutrition (MeSH) } \\
\text { 'Nutritional status' (MeSH) } \\
\text { 'Nutritional support' (MeSH) } \\
\text { 'Nutrition assessment' (MeSH) } \\
\text { 'Patient care planning' (MeSH) }\end{array}$ & $\begin{array}{l}\text { 'Transitional care' (MeSH) } \\
\text { 'Patient discharge' (MeSH) } \\
\text { 'Patient transfer' (MeSH) } \\
\text { 'Patient handoff' (MeSH) }\end{array}$ \\
\hline
\end{tabular}

*Identifies search terms with truncated keywords.

consider descriptive observational studies, including case series, individual case reports and descriptive cross-sectional studies, for inclusion.

Theses and dissertations, as well as reviews and systematic literature reviews of programmes that meet the inclusion criteria, will also be considered. The search strategy will aim to locate both published and unpublished studies. Conference papers will not be included.

The review will consider studies that include all adults (aged 18 years or older) who have been discharged from hospitals during the preceding 3 months. Adults of either sex and discharged from surgical, medical and rehabilitation wards during the preceding 3 months will be included. We will include studies lasting longer than 3 months if they report results of up to 3 months after discharge. There will be no language restrictions.

A three-step search strategy will be utilised in this review, as recommended by the Joanna Briggs Institute. ${ }^{26}$ The first strategical search step and terms will be chosen after discussion with a research librarian to retrieve the maximum number of articles. An initial limited search of MEDLINE via PubMed and CINAHL will be undertaken followed by an analysis of the text present in the title and abstract as well as of the index terms used to describe the article. A second search step using all the identified keywords and index terms will then be undertaken across all of the included databases, and citation searches will also be performed. Finally, in the third step, the reference lists of all the identified reports and articles will be screened for additional studies.

Databases will be searched from the date of their inception to the present date. If relevant, the reviewers will contact authors of the primary studies or reviews for further information.

\section{Stage 3: study selection}

The core concept of this scoping review is the effectiveness of individualised nutritional care plans. An individualised care plan is one that has been developed based on an individual assessment of patients' nutritional needs, conditions and desires. The plan entails appropriate nutritional interventions that will be evaluated and adjusted according to changes in the situation and condition of patients. ${ }^{26}$ The inclusion criteria of this study are that the patients obtain an individualised nutritional care plan during their hospital stay, and that the patient is followed-up for the next 3 months after discharge from the hospital.

This review will consider studies conducted among patients from all settings who were reviewed at any hospital during 3 months after discharge, and will not be limited to a particular geographic location or culture.

The literature search results will first be imported into Endnote as an .xml file and then uploaded to Covidence, an internet-based software programme that facilitates screening, data extraction and collaboration among reviewers.

Two authors will independently screen the titles and abstracts yielded by the search against the inclusion criteria. Full reports will be obtained for all titles that appear to meet the inclusion criteria or where there is any uncertainty. The same two authors will then screen the full-text reports and decide whether these meet the inclusion criteria.

\section{Step 4: charting the data}

The data will be independently charted from papers included in the scoping review by two of the authors. Differences in opinion will be resolved through discussion with each other or by consulting a third reviewer. A standardised charting form will be developed by the authors to allow the investigators to categorise the data. A draft charting form is provided in table 2. The draft data charting tool will be modified and revised as necessary while extracting data from each included study. Modifications will be detailed in the full scoping review article. 
Table 2 Data charting form (proposed)

\begin{tabular}{|c|c|c|c|c|c|c|c|c|}
\hline $\begin{array}{l}\text { Authors, } \\
\text { year, } \\
\text { country }\end{array}$ & $\begin{array}{l}\text { Participants } \\
\text { number and } \\
\text { setting }\end{array}$ & Methodology & $\begin{array}{l}\text { Aim } \\
\text { of the } \\
\text { study }\end{array}$ & $\begin{array}{l}\text { Study } \\
\text { design }\end{array}$ & Interventions & $\begin{array}{l}\text { Study } \\
\text { measurements }\end{array}$ & $\begin{array}{l}\text { Main } \\
\text { results/ } \\
\text { findings }\end{array}$ & $\begin{array}{l}\text { Authors } \\
\text { conclusion }\end{array}$ \\
\hline
\end{tabular}

\section{Stage 5: collating, summarising and reporting the results}

This scoping review will provide a narrative account of findings from the existing literature through thematic content analysis of the extracted literature. The data extracted from each of the studies will be mapped and presented in a form that logically reflects the objectives of this scoping review. Tabular and graphical representations of the data may be used to illustrate the identified results and will be supported with narrative descriptions of the data. The data from the studies will be presented and discussed in terms of the overall concepts/components that could be related to the effectiveness of individualised nutritional care plans for adults during hospital stay and the first 3 months after discharge.

\section{Patient and public involvement}

No patients are involved in the design and conception of this study.

\section{ETHICS AND DISSEMINATION}

Since all data will be obtained from publicly available materials, this study will not require any ethical approval. In terms of dissemination activities, an article reporting the results of the scoping review will be submitted for publication to an open-access peer-reviewed journal and presented at relevant conferences. We anticipate that the results of this review will provide a comprehensive overview of the field, which will help to guide nutritional practice and future research.

\section{Author affiliations}

${ }^{1}$ Faculty of Nursing and Health Sciences, Nord University—Levanger Campus, Levanger, Norway

${ }^{2}$ Faculty of Nursing and Health Sciences, Nord University-Bodo Campus, Bodo, Norway

${ }^{3}$ Medical Library, Aalborg University Hospital, Aalborg, Denmark

${ }^{4}$ Danish Centre of Clinical Guidelines and Danish Centre of Systematic Reviews, A Joanna Briggs Institute Centre of Excellence, Aalborg University, Aalborg, Denmark

Acknowledgements The authors wish to thank the Faculty of Nursing and Health Sciences, Nord University, that financed a course in scoping review.

Contributors Kl contributed to developing the research questions and methods, and contributed substantially to the drafting and editing. LU edited and provided feedback throughout the writing process. IGK commented on the protocol several times during the writing process. CS and PUP contributed to the development of the search strategy. PUP contributed to developing the research questions and was a major contributor to writing the protocol. All the authors read and approved the final version of the manuscript.

Funding KI, LU and IGK received funding from the Faculty of Nursing and Health Sciences, Nord University. CS and PP received funding from the Aalborg University Hospital.

Competing interests None declared.
Patient consent for publication Not required.

Provenance and peer review Not commissioned; externally peer reviewed.

Open access This is an open access article distributed in accordance with the Creative Commons Attribution Non Commercial (CC BY-NC 4.0) license, which permits others to distribute, remix, adapt, build upon this work non-commercially, and license their derivative works on different terms, provided the original work is properly cited, appropriate credit is given, any changes made indicated, and the use is non-commercial. See: http://creativecommons.org/licenses/by-nc/4.0/.

\section{REFERENCES}

1. Meijers JMM, Halfens RJG, van Bokhorst-de van der Schueren $\mathrm{MAE}$, et al. Malnutrition in Dutch health care: prevalence, prevention, treatment, and quality indicators. Nutrition 2009;25:512-9.

2. Elia M, Russell CA, Stratton RJ. Malnutrition in the UK: policies to address the problem. Proc Nutr Soc 2010;69:470-6.

3. Rasheed S, Woods RT. Predictive validity of 'Malnutrition Universal Screening Tool' ('MUST') and Short Form Mini Nutritional Assessment (MNA-SF) in terms of survival and length of hospital stay. Espen J 2013;8:e44-50.

4. Abd Aziz NAS, Mohd Fahmi Teng NI, Ab. Hamid MR, et al. Assessing the nutritional status of hospitalized elderly. Clin Interv Aging 2017;12:1615-25.

5. Kondrup J, Sorensen JM. The magnitude of the problem of malnutrition in Europe. Nestle Nutr Workshop Ser Clin Perform Programme 2009;12:1-14.

6. Allard JP, Keller H, Jeejeebhoy KN, et al. Malnutrition at hospital admission-contributors and effect on length of stay: a prospective cohort study from the Canadian malnutrition Task force. J Parenter Enteral Nutr 2016;40:487-97.

7. Allard JP, Keller H, Teterina A, et al. Factors associated with nutritional decline in hospitalised medical and surgical patients admitted for $7 \mathrm{D}$ or more: a prospective cohort study. Br J Nutr 2015;114:1612-22.

8. Stratton RJ, King CL, Stroud MA, et al. 'Malnutrition Universal Screening Tool' predicts mortality and length of hospital stay in acutely ill elderly. Br J Nutr 2006;95:325-30.

9. Caccialanza R, Klersy C, Cereda E, et al. Nutritional parameters associated with prolonged hospital stay among ambulatory adult patients. Can Med Assoc J 2010;182:1843-9.

10. Badgwell B, Stanley J, Chang GJ, et al. Comprehensive geriatric assessment of risk factors associated with adverse outcomes and resource utilization in cancer patients undergoing abdominal surgery. J Surg Oncol 2013;108:182-6.

11. Marshall S, Bauer J, Isenring $E$. The consequences of malnutrition following discharge from rehabilitation to the community: a systematic review of current evidence in older adults. $J$ Hum Nutr Diet 2014;27:133-41.

12. Bell JJ, Pulle RC, Crouch AM, et al. Impact of malnutrition on 12-month mortality following acute hip fracture. ANZ J Surg 2016;86:157-61.

13. Håkonsen SJ, Pedersen PU, Bjerrum M, et al. Nursing minimum data sets for documenting nutritional care for adults in primary healthcare: a scoping review. JBI Database System Rev Implement Rep 2018;16:117-39.

14. Organization for Economic Co-operation and Development. Health at a glance 2017: OECD indicators. Paris: OECD Publishing, 2017.

15. Organization for Economic Co-operation and Development. Length of hospital stay (indicator). Available: https://data.oecd.org/ healthcare/length-of-hospital-stay.htm [Accessed 1 Aug 2019].

16. Beck AM, Holst M, Rasmussen $\mathrm{HH}$. Oral nutritional support of older (65 years+) medical and surgical patients after discharge from Hospital: systematic review and meta-analysis of randomized controlled trials. Clin Rehabil 2013;27:19-27.

17. Beck A, Andersen UT, Leedo E, et al. Does adding a dietician to the liaison team after discharge of geriatric patients improve 
nutritional outcome: a randomised controlled trial. Clin Rehabil 2015:29:1117-28

18. Pedersen JL, Pedersen PU, Damsgaard EM. Nutritional follow-up after discharge of malnourished geriatric patients - design of a randomized clinical study. J Nutr Health Aging 2015;4:92-101.

19. Munk T, Tolstrup U, Beck AM, et al. Individualised dietary counselling for nutritionally at-risk older patients following discharge from acute hospital to home: a systematic review and meta-analysis. J Hum Nutr Diet 2016;29:196-208.

20. Allen J, Hutchinson AM, Brown R, et al. User experience and care integration in transitional care for older people from hospital to home. Qual Health Res 2017;27:24-36.

21. Prvu Bettger J, Alexander KP, Dolor RJ, et al. Transitional care after hospitalization for acute stroke or myocardial infarction. Ann Intern Med 2012;157:407-16.

22. Keller H, Payette $\mathrm{H}$, Laporte $\mathrm{M}$, et al. Patient-Reported dietetic care post Hospital for free-living patients: a Canadian malnutrition Task force study. J Hum Nutr Diet 2018;31:33-40.

23. Sharma $\mathrm{Y}$, Thompson $\mathrm{CH}$, Kaambwa B, et al. Investigation of the benefits of early malnutrition screening with telehealth follow up in elderly acute medical admissions. QJM-Int J Med 2017;110:639-47.

24. Ha L, Hauge T, Spenning AB, et al. Individual, nutritional support prevents undernutrition, increases muscle strength and improves QOL among elderly at nutritional risk hospitalized for acute stroke: a randomized, controlled trial. Clin Nutr 2010;29:567-73.
25. Lindegaard Pedersen J, Pedersen PU, Damsgaard EM. Nutritional follow-up after discharge prevents readmission to hospital - A randomized clinical trial. J Nutr Health Aging 2017;21:75-82.

26. Dorner B, Friedrich EK. Position of the Academy of nutrition and dietetics: individualized nutrition approaches for older adults: longterm care, post-acute care, and other settings. J Acad Nutr Diet 2018:118:724-35.

27. NSW Nutrition Care. Policy Directive. Nsw government health, 2017. Available: https://intranet.nnswlhd.health.nsw.gov.au/docs/PD2017 041-nutrition-care-v-002.pdf [Accessed 2 Aug 2019].

28. Peters M, Godfrey C, Mclnerney P, et al. Methodology for JBI scoping reviews. In: Aromataris E, ed. The Joanna Briggs Institute reviewers' manual 2015. Adelaide, Australia: The Joanna Briggs Institute, 2015: 1-24.

29. Munn Z, Peters MDJ, Stern C, et al. Systematic review or scoping review? guidance for authors when choosing between a systematic or scoping review approach. BMC Med Res Methodol 2018;18:143.

30. Arksey H, O'Malley L. Scoping studies: towards a methodological framework. Int J Soc Res Methodol 2005;8:19-32.

31. Levac D, Colquhoun H, O'Brien KK. Scoping studies: advancing the methodology. Implementation Sci 2010;5.

32. The Joanna Briggs Institute. Joanna Briggs Institute Reviewers' manual: 2017 edition, 2017. Available: https://reviewersmanual. joannabriggs.org/ [Accessed 3 Jan 2019].

33. Tricco AC, Lillie E, Zarin W, et al. PRISMA extension for scoping reviews (PRISMA-ScR): checklist and explanation. Ann Intern Med 2018;169:467-73. 